\title{
Duality and Bosonisation in Arbitrary Dimensions
}

\author{
R. Banerjee \\ S.N.Bose National Centre for Basic Sciences. \\ DB 17, Sector 1, Salt Lake City, Calcutta 700064, INDIA
}

\begin{abstract}
A functional integral approach is developed to discuss the bosonisation of the massive Thirring and the massive Schwinger models in arbitrary D-dimensions. It is found that these models, to all orders in the inverse fermi mass, bosonise to a theory involving a usual gauge field and a (D-2) rank antisymmetric (Kalb-Ramond) tensor field. Explicit bosonisation identities for the fermion current are deduced. Specialising to the lowest order reveals (for any $D \geq 4$ ) a mapping between the massive Thirring model and the Proca model. It also establishes an exact duality between the Proca model and the massive (D-2) rank Kalb-Ramond model. Schwinger terms in the current algebra are computed. Conventional bosonisation results in $\mathrm{D}=2,3$ are reproduced.
\end{abstract}




\section{Introduction}

The technique of bosonisation which consists in expressing a theory of fermions in terms of bosons provides a powerful nonperturbative tool for investigations in either quantum field theory [1] or condensed matter systems [2]. This idea of bosonisation has been recently extended in an interesting series of papers $[3,4]$ which reveal that the fermionic and bosonic versions of the theory are two extremes of a characterisation which, at intermediate stages, is a mixed representation of apparently interacting fermions and bosons. It is important to stress, however, that bosonisation (or its recent extension) is well established in only two (i.e. 1+1) space-time dimensions. This is because Schwinger terms which can give a clue to bosonisation are rather complicated in higher dimensions. Moreover, extracting bosonisation from a seemingly interacting theory of bosons and fermions requires the computation of the 1-cocycle for chiral transformations [5]. Apart from the fact that such transformations are meaningful only in even dimensions, it should also be realised that closed form expressions for the 1-cocycle are readily calculable only in two $(1+1)$ space-time dimensions. Inspite of these difficulties, some understanding of bosonisation in higher dimensions has been attained [6-10]. Specially, in $2+1$ dimensions, the bosonisation of the massive Thirring model to leading order in the inverse fermion mass has been performed [9]. The successful completion of this program by extending the computations to all orders has also been done [10]. In dimensions greater than $2+1$, however, the situation is rather obscure involving both technical and conceptual problems. In [7], the bosonisation of a massive fermion interacting with an external potential has been done but only upto the leading order in the inverse mass. The bosonised theory, moreover, is found to be nonlocal. Ref[8], on the other hand, considers the bosonisation of a free theory but the analysis is once again valid only upto the leading order in the inverse fermion mass.

In the present paper a functional integral approach is used to systematically discuss the bosonisation of fermionic theories, particularly the massive Thirring and Schwinger models, in any dimensions. The bosonised versions as well as the various bosonisation identities in the charge zero sector of the models are derived to all orders in the inverse fermion mass. Contrary to the findings reported in [7], all the boson-fermion identifications are local. Specifically, it has been shown that the massive Thirring model (MTM) in $D \geq 3$ space-time dimensions, to all orders in the inverse mass, is equivalent to a gauge theory involving a usual gauge field and a massless antisymmetric (D-2) rank tensor field. Similar conclusions also hold for the massive Schwinger model (MSM) except that the antisymmetric tensor field is massive. Specialising next to the leading $\left(\mathrm{m}^{-1}\right)$ order it is shown that for $D \geq 4$ the gauge theory representing the MTM simplifies to the Proca theory. Alternatively it can also be expressed in terms of a free massive (D-2) rank 
antisymmetric tensor field (massive Kalb-Ramond field). This approach to bosonisation therefore provides a duality between the Proca field and the massive Kalb-Ramond field in any dimensions $D \geq 4$. Incidentally the $D=3$ case may be recalled $[9,10]$ where the bosonisation of the MTM reproduces the well known mapping [11] between the self-dual model of [12] and the Maxwell-Chern-Simons theory [13]. This, as well as the familiar $D=2$ dimensional bosonisation [1] are easily reproduced. Finally, the leading order bosonisation of the MSM just yields the conventional Maxwell theory.

In section 2 the MTM is expressed as a gauge theory of apparently interacting fermions and bosons. This way of interpreting the MTM is reminiscent of the approach in [3] where the conventional two dimensional Thirring model is embedded in a bigger gauge theory by performing a chiral transformation. The role of gauge invariance and its connection to the dual (gauge invariant) version of MTM is elaborated. A corresponding construction for the MSM is also given. Section 3 discusses in detail the bosonisation of MTM and MSM. Furthermore, bosonisation identities mapping operators in the MTM and MSM with corresponding operators in the dual (bosonised)theories are given. The duality between the Proca model and the massive Kalb-Ramond model for any $D \geq 4$ is revealed in section 4 as a nontrivial application of the bosonisation program. As yet another application the explicit computation of Schwinger terms, to the leading $m^{-1}$ order, in the algebra of fermionic currents is performed. Some concluding remarks are presented in section 5 .

\section{Dualisation of fermionic theories}

In this section it is first shown how the MTM can be expressed in its dual form which turns out to be a gauge theory. Consider the lagrangian in $(D=d+1)$ space-time dimensions. 円

$\mathcal{L}=\bar{\psi}(i \not \partial-m-\lambda \not B) \psi+\frac{(-1)^{D}}{2(D-1)} F_{\mu_{1} \mu_{2} \ldots \mu_{D-1}} F^{\mu_{1} \mu_{2} \ldots \mu_{D-1}}+\epsilon_{\alpha \beta \mu_{1} \mu_{2} \ldots \mu_{D-2}} B^{\alpha} \partial^{\beta} A^{\mu_{1} \mu_{2} \ldots . \mu_{D-2}}$

where $A^{\mu_{1} \mu_{2} \ldots \mu_{D}}$ is an antisymmetric D rank tensor (the Kalb-Ramond field) and $F^{\mu_{1} \ldots . . \mu_{D+1}}$ is the corresponding field tensor,

$$
F^{\mu_{1} \ldots \mu_{D+1}}=\partial^{\left[\mu_{1}\right.} A^{\left.\mu_{2} \ldots \mu_{D+1}\right]}
$$

with the symbol [] denoting antisymmetrisation. The other field $B^{\mu}$ is an external vector field. This lagrangian is invariant under the independent gauge transformations,

$$
B_{\mu} \rightarrow B_{\mu}+\partial_{\mu} \omega ; \psi \rightarrow e^{-i \lambda \omega} \psi
$$

\footnotetext{
${ }^{1}$ The ensuing analysis is for any $D \geq 3$. The $\mathrm{D}=2$ case will be treated separately since it is special.
} 


$$
A_{\mu_{1} \ldots \mu_{D}} \rightarrow A_{\mu_{1} \ldots \mu_{D}}+\partial_{\left[\mu_{1}\right.} \Lambda_{\left.\mu_{2} \ldots \mu_{D}\right]}
$$

The equation of motion obtained by varying the distinct fields in (1) are found to be,

$$
\begin{gathered}
(i \not \partial-m-\lambda \not B) \psi=0 \\
\lambda j_{\mu}-\epsilon_{\mu \beta \mu_{1} \ldots \mu_{D-2}} \partial^{\beta} A^{\mu_{1} \ldots \mu_{D-2}}=0 ; j_{\mu}=\bar{\psi} \gamma_{\mu} \psi \\
\epsilon_{\alpha \beta \mu_{1} \ldots \mu_{D-2}} \partial^{\beta} B^{\alpha}+(-1)^{D} \partial^{\alpha} F_{\alpha \mu_{1} \ldots \mu_{D-2}}=0
\end{gathered}
$$

Since $B_{\mu}$ is an external field it may be eliminated at the classical level by using the equation (5). The dual transformation now consists in making further use of (5) to recast the field tensor in (1) in terms of the fermionic current,

$$
\lambda^{2} j_{\mu} j^{\mu}=\frac{(-1)^{D-1}(D-1) !}{(D-1)^{2}} F_{\mu_{1} \ldots \mu_{D-1}} F^{\mu_{1} \ldots \mu_{D-1}}
$$

Using this result the lagrangian (1) simplifies to,

$$
\mathcal{L}_{M T M}=\bar{\psi}(i \not \supset-m) \psi-\frac{\lambda^{2}}{2(D-2) !} j_{\mu} j^{\mu}
$$

which is just the lagrangian for the MTM. This is in fact the dual version of (1).

To verify whether this duality (which was a classical result) is preserved at the quantum level, it is necessary to work out the partition function corresponding to (1),

$$
\begin{aligned}
& Z=\int d\left[\psi, \bar{\psi}, B_{\mu}, A_{\mu_{1} \ldots \mu_{D-2}}\right] \delta\left(\partial_{\mu} B^{\mu}\right) \delta\left(\partial_{\mu_{1}} A^{\mu_{1} \ldots \mu_{D-2}}\right) \\
& \exp i \int\left(\mathcal{L}+\epsilon_{\mu \nu \mu_{1} \ldots \mu_{D-2}} \partial^{\nu} A^{\mu_{1} \ldots \mu_{D-2}} J^{\mu}+B_{\mu} K^{\mu}\right) d^{D} x
\end{aligned}
$$

where external sources $J_{\mu}, K_{\mu}$ coupled to the Kalb-Ramond field and $B_{\mu}$, respectively, have been introduced. Moreover to preserve gauge invariance of the action, the source $K_{\mu}$ must be conserved i.e. $\partial_{\mu} K^{\mu}=0$. The measure has been modified by inserting $\delta$ functions as the (Lorentz) gauge fixing conditions corresponding to the two independent gauge invariances (3). The Gaussian integration over the Kalb-Ramond field is easily performed by implementing the gauge $\partial_{\mu_{1}} A^{\mu_{1} \ldots \mu_{D-2}}=0 \mathrm{using}$ ' $\mathrm{t}$ Hooft's prescription to yield,

$$
\begin{aligned}
Z= & \int d\left[\psi, \bar{\psi}, B_{\mu}\right] \delta\left(\partial_{\mu} B^{\mu}\right) \exp i \int d^{D} x[\bar{\psi}(i \not \partial-m-\lambda \not B) \psi \\
& \left.+\frac{(D-2) !}{2}\left(B_{\mu}+J_{\mu}\right)^{2}+B_{\mu} K^{\mu}-\frac{(D-2) !}{2} J_{\alpha} \frac{\partial^{\alpha} \partial^{\beta}}{\square} J_{\beta}\right]
\end{aligned}
$$


Note the occurrence of a nonlocal term involving only the sources. This will be cancelled after the $B_{\mu}$ intergration is done. To see this express $\delta\left(\partial_{\mu} B^{\mu}\right)$ as a Fourier transform with variable $\beta(x)$. Then (10) may be written as,

$$
\begin{aligned}
Z= & \int d\left[\psi, \bar{\psi}, B_{\mu}, \beta\right] \exp i \int d^{D} x\left[\bar{\psi}\left(i \not \partial-m-\lambda\left(\not \beta+\frac{1}{(D-2) !} \not \partial \beta\right)\right) \psi\right. \\
& +\frac{(D-2) !}{2}\left\{\left(B_{\mu}+\frac{1}{(D-2) !} \partial_{\mu} \beta\right)^{2}+J_{\mu}^{2}+2 J^{\mu}\left(B_{\mu}+\frac{1}{(D-2) !} \partial_{\mu} \beta\right)\right\} \\
& -\frac{1}{2(D-2) !} \partial_{\mu} \beta \partial^{\mu} \beta-J^{\mu} \partial_{\mu} \beta+\left(B_{\mu}+\frac{1}{(D-2) !} \partial_{\mu} \beta\right) K^{\mu} \\
& \left.-\frac{(D-2) !}{2} J_{\alpha} \frac{\partial^{\alpha} \partial^{\beta}}{\square} J_{\beta}\right]
\end{aligned}
$$

where use has been made of the fact that $K_{\mu}$ is a conserved source and that the vector current $j_{\mu}=\bar{\psi} \gamma_{\mu} \psi$ is conserved. In other workds I shall always be considering some regularisation of the fermion determinant which preserves gauge invariance thereby retaining the classical conservation law $\partial_{\mu} j^{\mu}=0$ obtainable from (5). Note that a guage invariant regularisation also plays a key role in the recent discussions on bosonisation given in [3-5]. Introducing the new fields $G_{\mu}=B_{\mu}+\frac{1}{(D-2) !} \partial_{\mu} \beta$ and integrating over $\beta$ yields,

$$
\begin{aligned}
Z= & \int d\left[\psi, \bar{\psi}, G_{\mu}\right] \exp i \int d^{D} x[\bar{\psi}(i \not \partial-m-\lambda \phi) \psi \\
& \left.+\frac{(D-2) !}{2}\left\{G_{\mu}^{2}+J_{\mu}^{2}+2 J_{\mu} G^{\mu}\right\}+G_{\mu} K^{\mu}\right]
\end{aligned}
$$

Note that, as announced earlier, the nonlocal term has been precisely cancelled. Finally, integrating over $G_{\mu}$ leads to,

$Z=\int d[\psi, \bar{\psi}] \exp i \int d^{D} x\left[\bar{\psi}(i \not \partial-m) \psi-\frac{\lambda^{2}}{2(D-2) !} j_{\mu} j^{\mu}+\frac{\lambda}{(D-2) !} j_{\mu}\left((D-2) ! J^{\mu}+K^{\mu}\right)\right]$

where $j_{\mu}$ is the fermionic current (5) and a nonpropagating contact term has been dropped. Such terms will henceforth always be ignored. In the absence of sources it is seen that (13) represents the partition function for the MTM. The normalisation of the current-current interaction term also agrees with that obtained in (8) by a classical analysis. I have thus shown that (1) represents the lagrangian that is dual to the MTM. In the precise sense this duality should be understood as an equivalence between the partition functions (9) and (13). Furthermore, an inspection of the source terms leads to the following mappings between the Thirring current and the corresponding operators in the dual theory,

$$
\lambda j_{\mu} \leftrightarrow \epsilon_{\mu \nu \mu_{1} \ldots \mu_{D-2}} \partial^{\nu} A^{\mu_{1} \ldots \mu_{D-2}} \leftrightarrow(D-2) ! B_{\mu}
$$


These identifications are manifestations of the equations of motion $(5,6)$.

Likewise it is straightforward to construct the dual theory for the MSM. Replacing the kinetic term in (1) by a mass term, we obtain,

$$
\mathcal{L}^{\prime}=\bar{\psi}(i \not \partial-m-\lambda \not B) \psi-\frac{(-1)^{D}(D-2) !}{2} A_{\mu_{1} \ldots \mu_{D-2}} A^{\mu_{1} \ldots \mu_{D-2}}+\epsilon_{\alpha \beta \mu_{1} \ldots \mu_{D-2}} B^{\alpha} \partial^{\beta} A^{\mu_{1} \ldots \mu_{D-2}}
$$

The classical equations of motion are once again given by (4), (5) while (6) is modified to,

$$
(-1)^{D}(D-2) ! A_{\mu_{1} \ldots \mu_{D-2}}+\epsilon_{\alpha \beta \mu_{1} \ldots \mu_{D-2}} \partial^{\beta} B^{\alpha}=0
$$

The dual version of (15) is now easily obtained by eliminating the Kalb-Ramond field (which is no longer dynamical) using (16),

$$
\mathcal{L}_{M S M}=\bar{\psi}(i \not \partial-m-\lambda \not B) \psi-\frac{1}{4} B_{\mu \nu} B^{\mu \nu}
$$

where,

$$
B_{\mu \nu}=\partial_{\mu} B_{\nu}-\partial_{\nu} B_{\mu}
$$

This is just the lagrangian for the MSM. A more formal derivation follows by considering the partition function,

$$
\begin{aligned}
Z^{\prime}= & \int d\left[\psi, \bar{\psi}, B_{\mu}, A_{\mu_{1} \ldots \mu_{D-2}}\right] \delta\left(\partial_{\mu} B^{\mu}\right) \exp i \int\left(\mathcal{L}^{\prime}+\right. \\
& \left.\epsilon_{\mu \nu \mu_{1} \ldots \mu_{D-2}} \partial^{\nu} A^{\mu_{1} \ldots \mu_{D-2}} J^{\mu}+B_{\mu} K^{\mu}\right)
\end{aligned}
$$

where, as in the previous case,couplings with external sources $J_{\mu}, K_{\mu}$ have been included. A Lorentz gauge fixing delta function has been included in the measure. The second delta function appearing in (9) is absent here because (15) is no longer invariant under gauge transformations of the Kalb-Ramond field. Performing the Gaussian integration over $A_{\mu_{1} \ldots \mu_{D-2}}$ yields,

$$
\begin{aligned}
Z^{\prime}= & \int d\left[\psi, \bar{\psi}, B_{\mu}\right] \delta\left(\partial_{\mu} B^{\mu}\right) \exp i \int(\bar{\psi}(i \not \partial-m-\lambda \not B) \psi \\
& \left.-\frac{1}{4} B_{\mu \nu}^{2}+\partial^{\nu} B_{\nu \mu} J^{\mu}+B_{\mu} K^{\mu}\right)
\end{aligned}
$$

In the absence of sources, (20) represents the partition function of the MSM in the Lorentz gauge. This confirms the duality of (15) and (17). Comparison of the source terms in (19) and (20) yields the identification between the field strengths in the MSM and its dual version,

$$
\partial^{\nu} B_{\nu \mu} \leftrightarrow \epsilon_{\mu \nu \mu_{1} \ldots \mu_{D-2}} \partial^{\nu} A^{\mu_{1} \ldots \mu_{D-2}}
$$


which is also a manifestation of the equation of motion (16).

The analysis so far, as was particularly mentioned in the first footnote has been for any $D \geq 3$. For the special case of $\mathrm{D}=2$, the lagrangian (1) gets replaced by,

$$
\mathcal{L}=\bar{\psi}(i \not \partial-m-\lambda \not B) \psi+\frac{1}{2}\left(\partial_{\mu} \theta\right)^{2}+\epsilon_{\alpha \beta} B^{\alpha} \partial^{\beta} \theta
$$

The partition function in the presence of external sources is given by,

$$
Z=\int d\left[\psi, \bar{\psi}, B_{\mu}, \theta\right] \delta\left(\partial_{\mu} B^{\mu}\right) \exp i \int\left(\mathcal{L}+\epsilon_{\mu \nu} \partial^{\nu} \theta J^{\mu}+B_{\mu} K^{\mu}\right) d^{2} x
$$

Contrary to the case $D \geq 3$ (see (9)), the partition function for $\mathrm{D}=2$ has only one constraining delta function since the lagrangian (22) is invariant under only one gauge transformation (the first one in (3)). Following identical steps that led from (9) to (13) enables one to carry out the integrations over the Bose fields in (23) to yeild,

$$
Z=\int d[\psi, \bar{\psi}] \exp i \int d^{2} x\left[\bar{\psi}(i \not \partial-m) \psi-\frac{\lambda^{2}}{2} j_{\mu} j^{\mu}+\lambda j_{\mu}\left(J^{\mu}+K^{\mu}\right)\right]
$$

which is just the partition function for the MTM in the presence of external sources. In fact it agrees with the general form given in (13) for $\mathrm{D}=2$.

One can similarly show that in D $=2$ the theory dual to the MSM (17) is governed by the Lagrangian obtained by replacing the Kalb-Ramond field in (15) by a scalar,

$$
\mathcal{L}^{\prime}=\bar{\psi}(i \not \partial-m-\lambda \not B) \psi-\frac{1}{2} \theta^{2}+\epsilon_{\alpha \beta} B^{\alpha} \partial^{\beta} \theta
$$

Having established the duality of both the MTM and MSM for any $D \geq 2$ with their corresponding embedded versions, it is straightforward to analyse the bosonisation of these models. This will be presented in the next section.

\section{Bosonisation}

It is best to illustrate bosonisation by starting from the simplest example which is in D $=2$ and then proceeding to higher dimensions.

i) $\mathrm{D}=2$ dimensions : 
Let me consider the massless version in which case the Thirring model is known to be exactly solvable. The partition function follows from (24),

$$
Z=\int d[\psi, \bar{\psi}] \exp i \int d^{2} x\left[\bar{\psi} i \not \partial \psi-\frac{\lambda^{2}}{2} j_{\mu} j^{\mu}+\lambda j_{\mu}\left(J^{\mu}+K^{\mu}\right)\right]
$$

and the dual (embedded) version is obtained from (22) and (23),

$$
\begin{aligned}
Z= & \int d\left[\psi, \bar{\psi}, B_{\mu}, \theta\right] \delta\left(\partial_{\mu} B^{\mu}\right) \exp i \int\left[\bar{\psi}(i \not \partial-\lambda \not B) \psi+\frac{1}{2}\left(\partial_{\mu} \theta\right)^{2}\right. \\
& \left.+\epsilon_{\alpha \beta} B^{\alpha} \partial^{\beta} \theta+\epsilon_{\alpha \beta} \partial^{\beta} \theta J^{\alpha}+B_{\mu} K^{\mu}\right]
\end{aligned}
$$

To get the bosonised form of (26) the fermion integration in (27) is first performed. This a well known [14] expression obtained from a gauge invariant regularisation,

$$
\begin{aligned}
Z= & \int d\left[B_{\mu}, \theta\right] \delta\left(\partial_{\mu} B^{\mu}\right) \exp i \int\left[-\frac{\lambda^{2}}{2 \pi} B^{\mu}\left(g_{\mu \nu}-\frac{\partial_{\mu} \partial_{\nu}}{\square}\right) B^{\nu}+\frac{1}{2}\left(\partial_{\nu} \theta\right)^{2}\right. \\
& \left.+\epsilon_{\alpha \beta}\left(B^{\alpha}+J^{\alpha}\right) \partial^{\beta} \theta+B_{\mu} K^{\mu}\right] d^{2} x
\end{aligned}
$$

Implementing the gauge $\partial_{\mu} B^{\mu}=0$ using 't Hooft's prescription and carrying out the $B_{\mu}$-integration yields,

$$
Z=\int d \theta \exp i \int\left[\frac{1}{2}\left(1+\frac{\pi}{\lambda^{2}}\right)\left(\partial_{\mu} \theta\right)^{2}+\epsilon_{\mu \nu} \partial^{\nu} \theta\left(\frac{\pi}{\lambda^{2}} K^{\mu}+J^{\mu}\right)\right] d^{2} x
$$

It is thus seen how the massless Thirring model gets identified with a free massless scalar theory. Comparison of the source terms in (26) and (29) reveal the mapping between the Thirring current and the topological current in the scalar theory,

$$
\lambda j_{\mu} \leftrightarrow N \epsilon_{\mu \nu} \partial^{\nu} \theta
$$

modulo a normalisation $\mathrm{N}$ which is $\frac{\pi}{\lambda^{2}}$ or 1 . This is a manifestation of the familiar arbitrariness in the definition of the Thirring current. For $\lambda^{2}=\pi$ both definitions agree and the Thirring model maps identically to the massless free scalar theory with,

$$
j_{\mu} \leftrightarrow \frac{1}{\sqrt{\pi}} \epsilon_{\mu \nu} \partial^{\nu} \theta ; \bar{\psi} i \not \partial \psi \leftrightarrow \frac{1}{2}\left(\partial_{\mu} \theta\right)^{2}
$$

reproducing the well known identifications.

In a similar spirit consider the partition function for the massless Schwinger model $(20)$,

$$
Z^{\prime}=\int d\left[\psi, \bar{\psi}, B_{\mu}\right] \delta\left(\partial_{\mu} B^{\mu}\right) \exp i \int\left[\bar{\psi}(i \not \partial-\lambda \not B) \psi-\frac{1}{4} B_{\mu \nu} B^{\mu \nu}+\left(\partial^{\nu} B_{\nu \mu}\right) J^{\mu}\right]
$$


Its dual (embedded) version follows from (25) which is the 2-dimensional analogue of (19),

$$
Z^{\prime}=\int d\left[\psi, \bar{\psi}, B_{\mu}, \theta\right] \delta\left(\partial_{\mu} B^{\mu}\right) \exp i \int\left[\bar{\psi}(i \not \partial-\lambda \not B) \psi-\frac{\theta^{2}}{2}+\epsilon_{\mu \nu} B^{\mu} \partial^{\nu} \theta+\epsilon_{\mu \nu} J^{\mu} \partial^{\nu} \theta\right] d^{2} x
$$

Doing the fermionic intergration yields,

$$
Z^{\prime}=\int d\left[B_{\mu}, \theta\right] \delta\left(\partial_{\mu} B^{\mu}\right) \exp i \int\left[\frac{\lambda^{2}}{2 \pi} B^{\mu}\left(g_{\mu \nu}-\frac{\partial_{\mu} \partial_{\nu}}{\square}\right) B^{\nu}-\frac{\theta^{2}}{2}+\epsilon_{\mu \nu}\left(B^{\mu}+J^{\mu}\right) \partial^{\nu} \theta\right] d^{2} x
$$

Working out the $B_{\mu}$ integration by implementing the gauge $\partial_{\mu} B^{\mu}=0$ using 't Hooft's prescription leads to,

$$
Z^{\prime}=\int d \theta \exp i \int\left[\frac{\pi}{2 \lambda^{2}}\left(\partial_{\mu} \theta\right)^{2}-\frac{\theta^{2}}{2}+\epsilon_{\mu \nu} J^{\mu} \partial^{\nu} \theta\right] d^{2} x
$$

Scaling $\theta \rightarrow \frac{\lambda}{\sqrt{\pi}} \theta$ gives the desired structure,

$$
Z^{\prime}=\int d \theta \exp i \int\left[\frac{1}{2}\left(\partial_{\mu} \theta\right)^{2}-\frac{\lambda^{2}}{2 \pi} \theta^{2}+\frac{\lambda}{\sqrt{\pi}} \epsilon_{\mu \nu} J^{\mu} \partial^{\nu} \theta\right] d^{2} x
$$

This reproduces the result that the Schwinger model bosonises to a massive free scalar theory with mass $M=\frac{\lambda}{\sqrt{\pi}}$. The corresponding mapping between operators is given by,

$$
\partial^{\nu} B_{\nu \mu} \leftrightarrow M \epsilon_{\mu \nu} \partial^{\nu} \theta
$$

The analysis for the massive (either Thirring or Schwinger) models follows by computing the fermion determinant as a perturbative expansion about the massless case. Using the result of [15] one can reobtain the known [1, 14] equivalence of the MTM(MSM) with the massless (massive) sine- Gordon theory.

\section{ii) $\mathrm{D}=3$ dimensions:}

As has been illustrated the bosonised version is obtained by explicitly evaluating the fermion determinant. This is usually done by using a gauge invariant regularisation and the expression is, in general, non-local. Although an exact result does not exist, it is possible to perform the computations under certain approximations. In particular, an expansion in the large fermion mass limit is quite popular. The 3-dimensional example is rather special since the leading term in this expansion is given by the Chern-Simons three form. It should also be mentioned that subsequent analysis is valid only in the large mass limit. The partition function for the MTM follows from (13),

$$
Z=\int d[\psi, \bar{\psi}] \exp i \int d^{3} x\left[\bar{\psi}(i \not \partial-m) \psi-\frac{\lambda^{2}}{2} j_{\mu} j^{\mu}+\lambda j_{\mu}\left(J^{\mu}+K^{\mu}\right)\right]
$$


while its dual version is obtainable from (9),

$$
\begin{aligned}
Z= & \int d\left[\psi, \bar{\psi}, B_{\mu}, A_{\mu}\right] \delta\left(\partial_{\mu} B^{\mu}\right) \delta\left(\partial_{\mu} A^{\mu}\right) \exp i \int d^{3} x \\
& \left.\bar{\psi}(i \not \partial-m-\lambda \not B) \psi-\frac{1}{4} F_{\mu \nu} F^{\mu \nu}+\epsilon_{\mu \nu \rho} B^{\mu} \partial^{\nu} A^{\rho}+\epsilon_{\mu \nu \rho} J^{\mu} \partial^{\nu} A^{\rho}+B_{\mu} K^{\mu}\right]
\end{aligned}
$$

Computing the fermion determinant in (38) in inverse powers of $\mathrm{m}$ [16],

$$
\begin{aligned}
Z= & \int d\left[B_{\mu}, A_{\mu}\right] \delta\left(\partial_{\mu} B^{\mu}\right) \delta\left(\partial_{\mu} A^{\mu}\right) \exp i \int d^{3} x\left[-\frac{\lambda^{2}}{8 \pi} \epsilon_{\mu \nu \lambda} B^{\mu} \partial^{\nu} B^{\lambda}\right. \\
& +\frac{\lambda^{2}}{24 \pi m} B_{\mu \nu} B^{\mu \nu}+\ldots .-\frac{1}{4} F_{\mu \nu} F^{\mu \nu}+\epsilon_{\mu \nu \rho} B^{\mu} \partial^{\nu} A^{\rho} \\
& \left.+\epsilon_{\mu \nu \rho} J^{\mu} \partial^{\nu} A^{\rho}+B_{\mu} K^{\mu}\right]
\end{aligned}
$$

where the dots are the higher order Seeley co-effecients in the expansion of the fermion determinant. Thus the MTM, to all orders in $m^{-1}$, can be mapped on to a gauge theory (40) with the identifications,

$$
\lambda j_{\mu} \leftrightarrow \epsilon_{\mu \nu \rho} \partial^{\nu} A^{\rho} \leftrightarrow B_{\mu}
$$

The gauge theory (40) can be further simplified by performing the Gaussian $A_{\mu}$ integration,

$$
\begin{aligned}
Z= & \int d B_{\mu} \delta\left(\partial_{\mu} B^{\mu}\right) \exp i \int d^{3} x\left[-\frac{\lambda^{2}}{8 \pi} \epsilon_{\mu \nu \lambda} B^{\mu} \partial^{\nu} B^{\lambda}\right. \\
& \left.+\frac{\lambda^{2}}{24 \pi m} B_{\mu \nu} B^{\mu \nu}+\ldots .+\frac{1}{2}\left(B_{\mu}+J_{\mu}\right)^{2}+B_{\mu} K^{\mu}-\frac{1}{2} J_{\mu} \frac{\partial^{\mu} \partial^{\nu}}{\square} J_{\nu}\right]
\end{aligned}
$$

The nonlocal piece can be cancelled by following similar steps that led from (10) to (12). Expressing $\delta\left(\partial_{\mu} B^{\mu}\right)$ by its Fourier transform with variable $\beta$ and then doing the $\beta$ integration yields,

$Z=\int d G_{\mu} \exp i \int d^{3} x\left[\frac{1}{2} G_{\mu}^{2}-\frac{\lambda^{2}}{8 \pi} \epsilon_{\mu \nu \lambda} G^{\mu} \partial^{\nu} G^{\lambda}+\frac{\lambda^{2}}{24 \pi m}\left(\partial_{\mu} G_{\nu}-\partial_{\nu} G_{\mu}\right)^{2}+\ldots . .+G_{\mu}\left(J^{\mu}+K^{\mu}\right)\right]$

where new fields $G_{\mu}=B_{\mu}+\partial_{\mu} \beta$ have been introduced. Eq.(43) represents the complete bosonisation of the MTM.The dots are to be identified with the dots occurring in (40) while the Thirring current gets mapped with the basic field $G_{\mu}$,

$$
\lambda j_{\mu} \leftrightarrow G_{\mu}
$$

Note that $G_{\mu}$, in contrast to $B_{\mu}$ is a gauge invariant field. It is essentially the Stückelberg transformed $B_{\mu}$. 
Coming next to the MSM, note that its partition function is given by (20) while the dualised version is obtained from (19),

$$
\begin{aligned}
Z^{\prime}=\int d\left[\psi, \bar{\psi}, B_{\mu}, A_{\mu}\right] \delta\left(\partial_{\mu} B^{\mu}\right) \exp i \int d^{3} x[\bar{\psi}(i \not \partial- & -m-\lambda \not B) \psi+\frac{1}{2} A_{\mu}^{2}+\epsilon_{\alpha \beta \mu} B^{\alpha} \partial^{\beta} A^{\mu} \\
& \left.+\epsilon_{\alpha \beta \mu} J^{\alpha} \partial^{\beta} A^{\mu}+B_{\mu} K^{\mu}\right]
\end{aligned}
$$

Computing the fermion determinant as done in going from (38) to (40),

$$
\begin{aligned}
Z^{\prime}= & \int d\left[B_{\mu}, A_{\mu}\right] \delta\left(\partial_{\mu} B^{\mu}\right) \exp i \int d^{3} x\left[-\frac{\lambda^{2}}{8 \pi} \epsilon_{\mu \nu \lambda} B^{\mu} \partial^{\nu} B^{\lambda}+\frac{\lambda^{2}}{24 \pi m} B_{\mu \nu}^{2}+\ldots \ldots\right. \\
& \left.+\frac{1}{2} A_{\mu}^{2}+\epsilon_{\alpha \beta \mu}\left(B^{\alpha}+J^{\alpha}\right) \partial^{\beta} A^{\mu}+B_{\mu} K^{\mu}\right]
\end{aligned}
$$

Doing the $A_{\mu}$ integration yeilds,

$$
\begin{aligned}
Z^{\prime}= & \int d B_{\mu} \delta\left(\partial_{\mu} B^{\mu}\right) \exp i \int d^{3} x\left[-\frac{\lambda^{2}}{8 \pi} \epsilon_{\mu \nu \lambda} B^{\mu} \partial^{\nu} B^{\lambda}\right. \\
& \left.+\left(\frac{\lambda^{2}}{24 \pi m}-\frac{1}{4}\right) B_{\mu \nu}^{2}+\ldots . .+J^{\mu} \partial^{\nu} B_{\nu \mu}+B^{\mu} K_{\mu}\right]
\end{aligned}
$$

which gives the complete bosonised form of the MSM.

\section{iii) $D \geq 4$ dimensions :}

The main difference from the 3-dimensional example is that the leading term in the fermion determinant is a Maxwell piece instead of the Chern-Simons 3-form. This leads to a bosonisation which is (nontrivially) different from the 3-dimensional theory. Moreover, contrary to a recent finding [7] which analyses the bosonisation of a fermion coupled to an external potential in four dimensions, nonlocal terms never appear in my discussion.

The partition function for the MTM $(D \geq 4)$ follows from (13), while the dual version is given by (9). Computing the fermion determinant in (9) using a gauge invariant regularisation yields,

$$
\begin{aligned}
Z= & \int d\left[B_{\mu}, A_{\mu_{1} \ldots \mu_{D-2}}\right] \delta\left(\partial_{\mu} B^{\mu}\right) \delta\left(\partial_{\mu_{1}} A^{\mu_{1} \ldots \mu_{D-2}}\right) \exp i \int d^{D} x \\
& {\left[-n_{D} \lambda^{2} B_{\mu}\left(\square g^{\mu \nu}-\partial^{\mu} \partial^{\nu}\right) B_{\nu}+\ldots .+\frac{(-1)^{D}}{2(D-1)} F_{\mu_{1} \mu_{2} \ldots \mu_{D-1}} F^{\mu_{1} \mu_{2} \ldots \mu_{D-1}}\right.} \\
& \left.+\epsilon_{\mu \nu \mu_{1} \ldots \mu_{D-2}} B^{\mu} \partial^{\nu} A^{\mu_{1} \ldots \mu_{D-2}}+\epsilon_{\mu \nu \mu_{1} \ldots \mu_{D-2}} J^{\mu} \partial^{\nu} A^{\mu_{1} \ldots \mu_{D-2}}+B_{\mu} K^{\mu}\right]
\end{aligned}
$$

where only the leading term involving a dimension dependent normalisation $n_{D}$ has been explicitly written. The subsequent dots denote the other terms in the Seeley expansion of 
the fermion determinant. This gives the complete bosonised form of the MTM, valid to all orders in $m^{-1}$. The mapping between the Thirring current and the relevant operators in the bosonised theory (48) is just given by (14). It is now simple to perform the Gaussian integration over the Kalb-Ramond field. This yields,

$$
\begin{aligned}
Z=\int d B_{\mu} \delta\left(\partial_{\mu} B^{\mu}\right) \exp i \int d^{D} x\left[n_{D} \lambda^{2} B^{\mu \nu} B_{\mu \nu}\right. & +\ldots+\frac{(D-2) !}{2}\left(B_{\mu}+J_{\mu}\right)^{2} \\
& \left.-\frac{(D-2) !}{2} J_{\mu} \frac{\partial^{\nu} \partial^{\nu}}{\square} J_{\nu}+B_{\mu} K^{\mu}\right]
\end{aligned}
$$

As shown in earlier examples, the nonlocal term is eliminated by expressing $\delta\left(\partial_{\mu} B^{\mu}\right)$ by its Fourier transfer with variable $\alpha(x)$ and then doing the integration over $\alpha(x)$.It leads to a local result,

$Z=\int d G_{\mu} \exp i \int d^{D} x\left[n_{D} \lambda^{2}\left(\partial_{\mu} G_{\nu}-\partial_{\nu} G_{\mu}\right)^{2}+\ldots .+\frac{(D-2) !}{2} G_{\mu}^{2}+(D-2) ! G_{\mu} J^{\mu}+G_{\mu} K^{\mu}\right]$

where new fields $G_{\mu}=B_{\mu}+\frac{1}{(D-2) !} \partial_{\mu} \alpha$, obtained from $B_{\mu}$ by a Stückelberg transformation, were introduced. Eq.(50) gives another bosonised representation, valid to all orders in the inverse mass, for the MTM. The mapping between the Thirring current and the gauge invariant field $G_{\mu}$ is obtained by comparing the source terms in (13) and (49), respectively,

$$
\lambda j_{\mu} \leftrightarrow(D-2) ! G_{\mu}
$$

The final part of this section is devoted to discussing the MSM, whose partition function is given in (20). Computing the fermion determinant of its dual theory (19), one finds,

$$
\begin{aligned}
Z^{\prime}= & \int d\left[B_{\mu}, A_{\mu_{1} \ldots \mu_{D-2}}\right] \delta\left(\partial_{\mu} B^{\mu}\right) \exp i \int d^{D} x\left[n_{D} \lambda^{2} B_{\mu \nu} B^{\mu \nu}+\ldots .-\frac{(-1)^{D}(D-2) !}{2}\right. \\
& \left.A_{\mu_{1} \ldots \mu_{D-2}}^{2}+\epsilon_{\alpha \beta \mu_{1} \ldots \mu_{D-2}}\left(B^{\alpha}+J^{\alpha}\right) \partial^{\beta} A^{\mu_{1} \ldots \mu_{D-2}}+B_{\mu} K^{\mu}\right]
\end{aligned}
$$

which gives the bosonised form with the mapping (21). Performing the integration over the Kalb-Ramond field yields,

$$
Z^{\prime}=\int d B_{\mu} \delta\left(\partial_{\mu} B^{\mu}\right) \exp i \int d^{D} x\left[\left(n_{D} \lambda^{2}-\frac{1}{4}\right) B_{\mu \nu} B^{\mu \nu}+\ldots+\left(\partial_{\mu} B^{\mu \nu}\right) J_{\nu}+B_{\mu} K^{\mu}\right]
$$

This result can also be obtained directly by computing the fermion determinant from (20). 


\section{Applications}

It is worthwhile to discuss some applications of the generalised approach to bosonisation discussed in the preceding sections. The analysis done uptill now (for $D \geq 3$ ) is valid to all orders in the inverse fermi mass. Some interesting consequences follow if one restricts to the leading (lowest) order term.

\section{i) $\mathrm{D}=3$ space-time dimensions:}

The bosonised version of the MTM is given by (43). In the lowest (i.e.upto $\left(m^{-1}\right)$ ) order, this simplifies to,

$$
Z=\int d G_{\mu} \exp i \int d^{3} x\left[\frac{1}{2} G_{\mu}^{2}-\frac{\lambda^{2}}{8 \pi} \epsilon_{\mu \nu \lambda} G^{\mu} \partial^{\nu} G^{\lambda}+G_{\mu} J^{\mu}\right]
$$

where one of the sources $K_{\mu}$ has been dropped since it provides no additional information. In the absence of the source term, this is the partition function of the self-dual model of [12]. This correspondence between the MTM and the self dual model was obtained earlier in $[9,10]$. Note further that the mapping (44) remains unaltered. The Thirring current, therefore, obeys a self-dual equation analogous to $G_{\mu}$,

$$
j_{\mu}-\frac{\lambda^{2}}{4 \pi} \epsilon_{\mu \nu \lambda} \partial^{\nu} j^{\lambda}=0
$$

It is possible to give another bosonised form for the MTM by starting from (40). Performing the $B_{\mu}$ integration leads to,

$$
\begin{aligned}
Z= & \int d A_{\mu} \delta\left(\partial_{\mu} A^{\mu}\right) \exp i \int d^{3} x \\
& {\left[\frac{2 \pi}{\lambda^{2}} \epsilon_{\mu \nu \lambda} A^{\mu} \partial^{\nu} A^{\lambda}-\frac{1}{4} F_{\mu \nu} F^{\mu \nu}+\epsilon_{\mu \nu \rho} J^{\mu} \partial^{\nu} A^{\rho}\right] }
\end{aligned}
$$

In the absence of sources this is just the partition function for the Maxwell-Chern-Simons (MCS) theory in the Lorentz gauge. The equivalence of the MTM with this theory was previously explored in $[9,10]$. Comparing the source terms in (13) and (56) yields the mapping,

$$
\lambda\left(j_{\mu}\right)_{M T M} \leftrightarrow\left(\epsilon_{\mu \nu \rho} \partial^{\nu} A^{\rho}\right)_{M C S}
$$

Furthermore, since (54) and (56) are just dual descriptions of the same theory, namely the MTM in the leading $\mathrm{m}^{-1}$ expansion, these theories must be completely equivalent. This, incidentally, reproduces the well known [9-11] equivalence between the self-dual model (54) and the MCS theory (56) with the identification,

$$
\left(G_{\mu}\right)_{S e l f-d u a l} \leftrightarrow\left(\epsilon_{\mu \nu \lambda} \partial^{\nu} A^{\lambda}\right)_{M C S}
$$


Coming next to the MSM, observe that its bosonised version is given by (47). In the lowest order, this is the partition function for the MCS theory in the Lorentz gauge. But I have just shown that this theory is also the bosonised version of the MTM. Consequently, in the leading $m^{-1}$ approximation, the partiion functions for the MTM and the MSM become equivalent.

\section{ii) $D \geq 4$ space-time dimensions:}

The bosonised version of the MTM is given by (50). Considering the leading term only and suitably scaling the $G_{\mu}$-field clearly shows that the partition function corresponds to the massive Maxwell model or the Proca model. Likewise, the Thirring current gets mapped on to the Proca field by (51). Note furthermore from (50) that the sources $J_{\mu}, K_{\mu}$ are coupled identically to $G_{\mu}$ so that henceforth, without any loss of generality, one of these (namely $K_{\mu}$ ) will be dropped.

It is now possible to provide another bosonised version of the MTM by starting from the dual theory (48). Instead of integrating out the Kalb-Ramond field which led to (50), one integrates over the $B_{\mu}$-field. The result is,

$$
\begin{aligned}
Z= & \int d A_{\mu_{1} \ldots \mu_{D-2}} \delta\left(\partial_{\mu_{1}} A^{\mu_{1} \ldots \mu_{D-2}}\right) \exp i \int d^{D} x\left[\frac{(1)^{D}}{2(D-1)} F_{\mu_{1} \mu_{2} \ldots \mu_{D-1}} F^{\mu_{1} \ldots \mu_{D-1}}\right. \\
& \left.+\frac{(-1)^{D}(D-2) !}{4 n_{D} \lambda^{2}} A_{\mu_{1} \ldots \mu_{D-2}} A^{\mu_{1} \ldots \mu_{D-2}}+\epsilon_{\mu \nu \mu_{1} \ldots \mu_{D-2}} J^{\mu} \partial^{\nu} A^{\mu_{1} \ldots \mu_{D-2}}\right]
\end{aligned}
$$

where the delta function prevents the occurrence of any non-local terms. As usual, the delta function may now be expressed by its Fourier transform and new fields $A_{\mu_{1} \ldots \mu_{D-2}}^{\prime} \rightarrow$ $A_{\mu_{1} \ldots \mu_{D-2}}+\partial_{\left[\mu_{1}\right.} \Lambda_{\left.\mu_{2} \ldots \mu_{D-2}\right]}$ are introduced where $\Lambda_{\mu_{2} \ldots \mu_{D-2}}$ is the Fourier variable. The integration over this variable, which decouples from the primed field, yields a trivial normalisation so that the final result simplifies to,

$$
\begin{aligned}
Z= & \int d A_{\mu_{1} \ldots \mu_{D-2}}^{\prime} \exp i \int d^{D} x\left[\frac{(-1)^{D}}{2(D-1)} F_{\mu_{1} \ldots \mu_{D-1}}\left(A^{\prime}\right) F^{\mu_{1} \ldots \mu_{D-1}}\left(A^{\prime}\right)\right. \\
& \left.+\frac{(-1)^{D}(D-2) !}{4 n_{D} \lambda^{2}} A_{\mu_{1} \ldots \mu_{D-2}}^{\prime} A^{\prime \mu_{1} \ldots \mu_{D-2}}+\epsilon_{\mu \nu \mu_{1} \ldots \mu_{D-2}} J^{\mu} \partial^{\nu} A^{\prime} \mu_{1} \ldots \mu_{D-2}\right]
\end{aligned}
$$

This shows that the MTM gets identified with the theory of a massive (D-2) rank Kalb-Ramond field. Furthermore, comparing the source terms in (13) and (60) gives the bosonisation rule for the Thirring current,

$$
\lambda j_{\mu} \leftrightarrow \epsilon_{\mu \nu \mu_{1} \ldots \mu_{D-2}} \partial^{\nu} A^{\prime} \mu_{1} \ldots \mu_{D-2}
$$


An important consequence of the above analysis is that, since (50) and (60) have a common origin (namely, the MTM in the leading $m^{-1}$ expansion), the partition functions represented by them must be exactly equivalent. This establishes the duality between the Proca model and the Kalb-Ramond model involving a massive (D-2) rank antisymmetric gauge field. The basic fields in these models are related by,

$$
(D-2) ! G_{\mu} \leftrightarrow \epsilon_{\mu \nu \mu_{1} \ldots \mu_{D-2}} \partial^{\nu} A^{\prime} \mu_{1} \ldots \mu_{D-2}
$$

Incidentally these findings may be regarded as the generalisation of the $\mathrm{D}=3$ case where a self dual model is identified with the Maxwell-Chern-Simons theory [9-11]. For the particular case of $\mathrm{D}=4$ only, a mapping similar to the one discussed here was reported elsewhere, though in a different context [17].

It is instructive to make a counting of the number of degrees of freedom in the two theories. The number of independent degrees of freedom for the Proca field in D dimensions is (D-1). Now the number of components of a (D-2) rank antisymmetric tensor in D dimensions is $\frac{D(D-1)}{2}$. Because of constraints all of these components are not independent. It is simple to verify that there are (D-1)(D-2) second class (phase-space) constriants. Hence the number of independent degrees of freedom is $\frac{1}{2}[D(D-1)-(D-1)(D-2)]=D-1$ and agrees with the number in the Proca theory.

\section{Schwinger terms}

It is well known that a conventional way [14] to discuss bosonisation is to start from Schwinger terms in the algebra of fermionic currents. Since Schwinger terms are difficult to compute in higher dimensions it reveals one of the reasons why bosonisation in these dimensions, by conventional techniques, becomes problematic. Here I shall adopt the reverse route whereby, knowing the bosonisation relations in arbitrary dimensions, the Schwinger terms in the current algebra 2 of fermionic theories will be evluated.

The $\mathrm{D}=2$ example is simple. Using (31) the current algebra in the Thirring model follows trivially,

$$
\begin{aligned}
i\left[j_{0}(x), j_{0}(y)\right] & =0 \\
i\left[j_{0}(x), j_{1}(y)\right] & =-\frac{1}{\pi} \partial_{1} \delta(x-y)
\end{aligned}
$$

as a consequence of the equal time algebra of the free scalar field.

\footnotetext{
${ }^{2}$ This algebra is always implied at equal time
} 
The $\mathrm{D}=2$ example is special since bosonisation is exact. In higher dimensions bosonisation has been achieved for massive fermionic theories only in the $m^{-1}$ approximation. In the subsequent analysis, moreover, I concentrate only on the leading order approximation. In $\mathrm{D}=3$ dimensions the MTM is mapped on to the MCS theory (56) with the identification (57). The components of the current correspond to the electric and magnetic fields in the MCS theory. Since the Poisson algebra among the latter fields is known [11], it is straightforward to compute the current algebra,

$$
\begin{aligned}
i\left[j_{0}(x), j_{0}(y)\right] & =0 \\
i\left[j_{0}(x), j_{i}(y)\right] & =-\frac{1}{\lambda^{2}} \partial_{i} \delta(x-y) \\
i\left[j_{l}(x), j_{m}(y)\right] & =\frac{4 \pi}{\lambda^{4}} \epsilon_{l m} \delta(x-y)
\end{aligned}
$$

and agrees with the result derived previously in [10].

The last part is devoted to the $D \geq 4$ case. Here the bosonised form of the MTM corresponds to the Proca theory (50) with the mapping (51). The current algebra is, therefore, obtainable by the algebra of the basic fields $G_{\mu}$ in the Proca theory. Since the Proca theory is a second class system, the Poisson algebra gets replaced by the Dirac algebra [18],

$$
\begin{aligned}
i\left[G_{0}(x), G_{0}(y)\right]_{D B} & =0 \\
i\left[G_{0}(x), G_{i}(y)\right]_{D B} & =-\frac{1}{(D-2) !} \partial_{i} \delta(x-y)
\end{aligned}
$$

where the suffix 'DB' denotes Dirac brackets. Using (51) and (65), the relevant current algebra follows,

$$
\begin{aligned}
& i\left[j_{0}(x), j_{0}(y)\right]=0 \\
& i\left[j_{0}(x), j_{i}(y)\right]=-\frac{(D-2) !}{\lambda^{2}} \partial_{i} \delta(x-y)
\end{aligned}
$$

Equations (63), (64) and (66) reveal that the Schwinger terms have an identical structure.

\section{Conclusions}

A systematic way of bosonising fermionic models in arbitrary dimensions has been developed. The basic idea was to use gauge invariance as a guiding principle to construct a 
parent lagrangian comprising both fermionic and bosonic fields. The partition function for this parent lagrangian in the presence of external sources was constructed with the measure suitably modified by gauge fixing delta functions. The bosonic integration was exactly carried out leading to some fermionic theory like the massive Thirring model (MTM) or the massive Schwinger model (MSM). Incidentally the structure of the fermionic models were identical to what one would expect by performing a duality transformation of the parent lagrangian on the classical level. Now instead of integrating out the bosonic fields, if the fermionic fields were integrated, the bosonised versions of the fermionic models were obtained. Furthermore, by comparing the source terms, explicit bosonisation identities for the fermionic currents were derived. The fermionic integration really implies the evaluation of the fermion determinant. Apart from $\mathrm{D}=2$ dimensional case, this result is not exactly known. It can be computed only in some approximation as for example the $m^{-1}$ expansion, where $\mathrm{m}$ is the fermion mass. Thus the bosonisation as well as the mappings between the various operators were valid for any $D \geq 3$ only in the large $m$ expansion. Moreover, contrary to other approaches [7-9] which discuss the bosonisation of only the free theory or that in the presence of an external field, the analysis presented here is valid to all orders in this inverse mass expansion.

It was shown in $D \geq 3$ that the partition functions for the MTM and MSM can be mapped on to the partition functions for a gauge theory involving two gauge fields - a usual vector field and an antisymmetric (D - 2) rank tensor field which is also called the Kalb-Ramond field. The fermion current, likewise, gets identified with the topological currents in the gauge theory. Furthermore, integrating over the Kalb-Ramond field resulted in an equivalent bosonised expression involving only the vector potential. All these computations were valid to arbitrary orders in the inverse fermi mass. Simplifying the computations to the leading $\left(0\left(\mathrm{~m}^{-1}\right)\right)$ order only, it was possible instead to perform the integration over the vector potential which led to a bosonised theory involving only the Kalb Ramond field. In this way it was found that, in the leading order, the MTM in D = 3 dimensions bosonises to the self dual model [12] or to the equivalent Maxwell-ChernSimons theory [13]. Similar conclusions were valid for the ( $D=3$ dimensional) MSM. For $D \geq 4$, on the other hand, the MTM was mapped on to the Proca model or its dual, which was found to be the theory of a massive (D-2) rank antisymmetric tensor field. The bosonisation technique developed here therefore provided an elegant way of discussing the duality between different bosonic theories. Incidentally, the duality between the self dual model and the Maxwell-Chern-Simons theory in D $=3$ or that between the Proca model and the second rank massive Kalb Ramond field in $\mathrm{D}=4$ are well known results [9-11, 17]. The present approach extends this duality for any D-dimension. Another application was to derive the Schwinger terms in the commutator algebra of fermionic currents by using the bosonisation relations. Moreover all the usual results in $D=2$ bosonisation 
were easily reproduced.

To put this work into proper perspective, a comparison with other recent approaches to bosonisation is relevant. The 'smooth bosonisation' approach [3, 4], which is primarily geared for $\mathrm{D}=2$ dimensions, uses different gauge fixing conditions in an embedded theory to recover either the original fermionic theory or its bosonised form. The present analysis, on the contrary, uses the same gauge condition but reverses the order of integration in the parent theory (which mimics the role of the embedded theory) to reproduce once, the fermionic theory or, alternatively, the corresponding bosonic theory. This is somewhat akin to the approach by using duality transformations [7, 19]. However, the full gauge freedom is not exploited there [7] leading to nonlocal terms in higher dimensional bosonisation. Such terms never occur in my analysis. Finally, the $\mathrm{D}=3$ dimensional appraoch of [9] uses as a parent lagrangian the expression suggested in [11] which is suitable for discussing bosonisation only in the leading $\left(\mathrm{m}^{-1}\right)$ order expansion. Significantly, all these approaches including the present one rely on the expression of the fermion determinant evaluated in the large-m approximation. It is clear, therefore, that different routes to bosonisation are connected - a fact which has also been realised in [8] - and this analysis provided fresh insights into this connection, apart from yielding new results in higher dimensions. 


\section{References}

[1] S. Coleman, Phys. Rev.D11 (1975) 2088; S. Mandelstam, ibid 3026.

[2] E. Lieb and D. Mattis, Jour. Math. Phys. 6 (1965) 304; A. Luther and I. Peschel, Phys. Rev. B12 (1975) 3908.

[3] P.H. Damgaard, H.B. Nielsen and R. Sollacher, Nucl. Phys. B385 (1992) 227; B414 (1994) 541; Phys.Lett. B296 (1992) 132; B322 (1994) 131.

[4] P.H. Damgaard and R. Sollacher, Nucl. Phys. B433 (1995) 671.

[5] N. Banerjee and R. Banerjee, Nucl. Phys. B445 (1995) 516.

[6] E.C. Marino, Phys. Lett. B263 (1991) 63; L. Huerta and J. Zanelli, Phys. Rev. Lett. 71 (1993) 3622;

A. Kovner and P.S. Kurzepa, Phys. Lett. B321 (1994) 129;

A.H. Castro Neto and E.H. Fradkin, Phys. Rev. Lett. 72 (1994) 1393.

[7] C.P. Burgess, C.A. Lütken and F. Quevedo, Phys. Lett. B336 (1994) 18.

[8] F.A. Schaposnik, Phys. Lett. B356 (1995) 39.

[9] E. Fradkin and F.A. Schaposnik, Phys. Lett. B338 (1994) 253.

[10] R. Banerjee, Phys. Lett. B358 (1995) 297.

[11] S. Deser and R. Jackiw, Phys. Lett. B139 (1984) 371;

R. Banerjee and H.J. Rothe, Nucl Phys. B447 (1995) 183.

[12] P.K. Townsend, K. Pilch and P.van Nieuwenhuizen, Phys. Lett. B136 (1984) 38.

[13] S. Deser, R. Jackiw and S. Templeton, Phys. Rev. Lett. 48 (1982) 975; Ann. Phys. 140 (1982) 372.

[14] E. Abdalla, M.C.B. Abdalla and K.D. Rothe, "Non-perturbative Methods in 2Dimensional Quantum Field Theory" (World Scientific, Singapore, 1991)

[15] H. Dorn, Phys. Lett. B167 (1986) 86.

[16] S. Deser and A. Redlich, Phys. Rev. Lett. 61 (1988) 1541.

[17] E. Cremmer and J. Scherk, Nucl. Phys. B72 (1974) 117;

T.J. Allen, M.J. Bowick and A. Lahiri, Mod. Phys. Lett. A6 (1991) 559. 
[18] N. Banerjee, R. Banerjee and S. Ghosh, SINP Report 1994 (to appear in Ann. Phys 1995).

[19] C.P. Burgess and F. Quevedo, Nucl. Phys. B421 (1994) 373. 